\title{
Lung cancer in Europe: The way forward
}

\author{
Monica Racovita', Eleanor Wheeler', Suzanne Wait', Dani Bancroft', Rowan Eastabrook', Tit Albrehtt, ${ }^{2,3}$ Anne-Marie Baird ${ }^{4,5}$, \\ Jacek Jassem ${ }^{6}$, Aoife McNamara ${ }^{7,}$, Silvia Novello9, ${ }^{90}$, Cornel Radu-Loghin" ${ }^{11}$, Jan P. van Meerbeeck ${ }^{12,13,14}$
}

Lung cancer causes more deaths than any other cancer, but for too long, it has not been given the policy attention it deserves. Lung cancer is the main cause of cancer-related deaths in Europe ${ }^{1}$, and its burden will remain high for several decades to come. Even though smoking rates are gradually declining ${ }^{2}$, people who used to smoke remain at high risk of lung cancer for many years after quitting $^{3}$, and environmental factors such as air pollution are a growing cause of lung cancer in the entire population ${ }^{4}$.

We have the means to reduce the burden of lung cancer on our society, but it will require a comprehensive and integrated approach. Importantly, we need to dispel the perception that lung cancer is a self-inflicted condition. We could start by treating smoking as an addiction and reducing stigma towards both smoking and lung cancer. Early detection needs to be enhanced by implementing largescale screening programmes that target high-risk individuals, alongside smoking cessation programmes. The evidence is clear that screening high-risk individuals using low-dose computed tomography scans offers a safe and effective way to shift diagnosis to earlier stages and reduce mortality from lung cancer ${ }^{5,6}$. Complementary approaches, such as incidental pulmonary nodule identification, management protocols and rapid referral pathways from primary to secondary care, are also important to improve early detection ${ }^{7,8}$. Improvements to lung cancer care pathways are needed to ensure all people have access to multidisciplinary care that encompasses specialist diagnosis, personalised treatments, and palliative and survivorship care ${ }^{7,8}$. Finally, targeted efforts are required to reduce geographical and socioeconomic disparities in access and outcomes ${ }^{9,10}$.

Making these changes can deliver benefits beyond lung cancer. Targeted screening programmes offer the opportunity for early detection of other non-communicable diseases (NCDs), such as chronic obstructive pulmonary disease and some forms of heart disease ${ }^{11-13}$. Tackling lung cancer can help countries progress towards reducing the overall burden of NCDs on their societies and contribute to greater health system sustainability.

With the implementation of Europe's Beating Cancer Plan and as we look to post-COVID recovery, we have the opportunity to put in place what is needed to improve outcomes for people with lung cancer and reduce the burden the condition poses on our societies. Lung cancer has, for too long, been denied due attention as a public health priority. The time to act is now.

\section{REFERENCES}

1. Ferlay J, Ervik M, Lam F, et al. Global Cancer Observatory: Cancer Today. International Agency for Research on Cancer; 2020. Updated December 1, 2020. Accessed November 25, 2021. https://gco.iarc.fr/today

2. Islami F, Torre LA, Jemal A. Global trends of lung cancer mortality and smoking

\section{AFFILIATION}

1 The Health Policy Partnership, London, United Kingdom 2 Department of Public Health, Medical Faculty, University of Ljubljana, Ljubljana, Slovenia 3 National Institute of Public Health of Slovenia, Ljubljana, Slovenia 4 Lung Cancer Europe, Bern, Switzerland

5 School of Medicine, Trinity Translational Medicine Institute, Trinity College Dublin, Dublin, Ireland 6 Medical University of Gdask, Gdask, Poland

7 Global Lung Cancer Coalition,

Dublin, Ireland

8 Irish Cancer Society, Dublin, Ireland 9 Women Against Lung Cancer in Europe, Turin, Italy 10 Department of Oncology, San Luigi Hospital, University of Turin, Turin, Italy

11 European Network for Smoking and Tobacco Prevention, Brussels, Belgium

12 European Cancer Organisation, Brussels, Belgium

13 European Respiratory Society, Lausanne, Switzerland

14 Oncology Department, Antwerp University Hospital, Antwerp, Belgium

\section{CORRESPONDENCE TO}

Monica Racovita. The Health Policy Partnership, 69 St Martin's Ln, WC2N 4JS, London, United Kingdom. E-mail: monica.racovita@hpolicy.com

Cornel Radu-Loghin. European Network for Smoking and Tobacco Prevention, Chau. d'Ixelles 144, 1050, Brussels, Belgium.

E-mail: cornel.radu@ensp.org

\section{KEYWORDS}

lung cancer, screening, treatment, Europe, COVID-19, noncommunicable diseases

Received: 11 February 2022 Accepted: 14 February 2022 
prevalence. Transl Lung Cancer Res. 2015;4(4):327-338. doi:10.3978/j.issn.2218-6751.2015.08.04

3. Tindle HA, Stevenson Duncan M, Greevy RA, et al. Lifetime Smoking History and Risk of Lung Cancer: Results From the Framingham Heart Study. J Natl Cancer Inst. 2018;110(11):1201-1207. doi:10.1093/jnci/djy041

4. Myers R, Brauer M, Dummer T, et al. High-Ambient Air Pollution Exposure Among Never Smokers Versus Ever Smokers With Lung Cancer. J Thorac Oncol. 2021;16(11):1850-1858. doi:10.1016/j.jtho.2021.06.015

5. de Koning HJ, van der Aalst CM, de Jong PA, et al. Reduced Lung-Cancer Mortality with Volume CT Screening in a Randomized Trial. N Engl J Med. 2020;382(6):503-513. doi:10.1056/NEJMoa1911793

6. National Lung Screening Trial Research Team. Reduced lung-cancer mortality with low-dose computed tomographic screening. N Engl J Med. 2011;365(5):395409. doi:10.1056/NEJMoa1102873

7. Lam S, Tammemagi M. Contemporary issues in the implementation of lung cancer screening. Eur Respir Rev. 2021;30(161):200288. doi:10.1183/16000617.0288-2020

8. Pollock M, Craig R, Chojecki D, et al. Initiatives to Accelerate the Diagnostic Phase of Cancer Care: An Environmental Scan. Institute of Health Economics; 2018.

9. Public Health England. PHE screening inequalities strategy. Updated October 22, 2020. Accessed November 25, 2021. https://www.gov.uk/government/publications/ nhs-population-screening-inequalities-strategy/phescreening-inequalities-strategy

10. Ali N, Lifford KJ, Carter B, et al. Barriers to uptake among high-risk individuals declining participation in lung cancer screening: a mixed methods analysis of the UK Lung Cancer Screening (UKLS) trial. BMJ Open. 2015;5(7):e008254. doi:10.1136/bmjopen-2015-008254

11. Reiter MJ, Nemesure A, Madu E, Reagan L, Plank A. Frequency and distribution of incidental findings deemed appropriate for S modifier designation on low-dose CT in a lung cancer screening program. Lung Cancer. 2018;120:1-6. doi:10.1016/j.lungcan.2018.03.017

12. Ruparel M, Quaife SL, Dickson JL, et al. Evaluation of cardiovascular risk in a lung cancer screening cohort. Thorax. 2019;74(12):1140-1146. doi:10.1136/thoraxjnl-2018-212812

13. Ruparel M, Quaife SL, Dickson JL, et al. Prevalence, Symptom Burden, and Underdiagnosis of Chronic Obstructive Pulmonary Disease in a Lung Cancer Screening Cohort. Ann Am Thorac Soc. 2020;17(7):869878. doi:10.1513/AnnalsATS.201911-8570C
CONFLICTS OF INTEREST

The authors have each completed and submitted an ICMJE Form for Disclosure of Potential Conflicts of Interest. M. Racovita, E. Wheeler, S. Wait, D. Bancroft, and R. Eastabrook (The Health Policy Partnership) report fees from AstraZeneca, Johnson \& Johnson, Elekta, and Philip , related to the current work, and funds from Amgen, AstraZeneca, Bristol Myers Squibb (BMS), Johnson \&t Johnson, Novartis, Advanced Accelerator Applications, Roche Diagnostics, Sanofi, GE Healthcare, Elekta, Vifor Pharma, UCB, Pfizer, Boehringer Ingelheim, and Philips, outside the submitted work. J. Jassem reports fees from AstraZeneca, MSD, and Exact Sciences, outside the submitted work. S. Novello reports consulting fees from BMS, Eli LILLY, Takeda, ROCHE, Pfizer, Astrazeneca, BI, MSD, Abbvie, Pharmamar, and Beigene, outside the submitted work. A. M. Braid reports consulting fees from Lungevity and honoraria from Roche, outside the submitted work. The rest of the authors have nothing to disclose.

\section{FUNDING}

This study is jointly funded by AstraZeneca, Johnson \& Johnson, Philips and Elekta. All outputs are non-promotional, evidence-based and shaped by the authors, who had editorial control and provided their time for free.

\section{ETHICAL APPROVAL AND INFORMED CONSENT}

Ethical approval and informed consent were not required for this study.

DATA AVAILABILITY

Data sharing is not applicable to this article as no new data were created.

PROVENANCE AND PEER REVIEW

Commissioned; internally peer reviewed.

\section{DISCLAIMER}

The views and opinions expressed in this article are those of the authors. 